\section{Infrapatellar fat pad maximal area and changes in knee symptoms: gender-related difference or gender difference in reporting?}

We read with interest a recent paper by Pan et al on the relationship between the infrapatellar fat pad (IPFP) and knee osteoarthritis (OA). ${ }^{1}$ With an average follow-up of 2.6 years, the authors found that IPFP maximal area at baseline was negatively associated with changes in knee pain, but positively associated with change in tibial cartilage volume per annum, and reduced risks of increases in medial cartilage defects. Based on these results, the authors concluded that a higher IPFP maximal area appears to have a protective role for knee symptoms and cartilage damage in older female adults.

We commend the authors for performing the first longitudinal study on the associations between IPFP size at baseline and changes in knee OA measures in community-based older adults. However, we would like to raise several concerns. First, the overall response rate was $57 \%, 25 \%$ of which were lost to follow-up. Since the assessment of knee pain was self-reported and change in knee pain was calculated as follow-up value minus the baseline value, it is possible that people with pain to start with were more likely to respond and those with significant changes in pain symptoms over the study interval were more likely to follow up. The authors stated that there were no significant differences in demographics and the knee cartilage volumes between subjects with and without the second MRI. However, the difference in demographics was not investigated between those with follow-up and without.

Second, the gender-related differences may be exaggerated given the subjective nature of patient's self-reported pain. Population-based research has consistently demonstrated greater pain prevalence among women relative to men. ${ }^{2}$ For example, large-scale epidemiological studies across multiple geographic regions show that pain is reported more frequently by women than men. ${ }^{3}$ In addition, data for cartilage volume loss and cartilage defects were also inconsistent. The baseline IPFP maximal area was not found to be significantly associated with change in patellar cartilage volume or increases in cartilage defects at the lateral tibial and femoral and patellar sites in women. There is no anatomical explanation for why this would be the case. These findings suggest that the difference in results between genders may be related to women's lower threshold for reporting pain.

Third, the authors stated that reducing IPFP area in the process of knee surgery may increase later knee pain in women. IPFP resection may not have any clinical impact in patients with severe OA, most of whom undergo total knee arthroplasty (TKA). Meta-analysis showed that for OA, there were no differences in function, range of motion and anterior knee pain between patients who underwent TKA with IPFP resection and those without. ${ }^{4}$ In a small randomised controlled trial, Pinsornsak et al found no significant differences in patellar tendon shortening, knee society score, functional score or knee flexion between patients who underwent minimally invasive TKA with IPFP excision and those without. ${ }^{5}$ However, IPFP resection resulted in a small percentage of patients with anterior knee pain after surgery. ${ }^{5}$ Consequently, IPFP resection should only be avoided if possible in patients undergoing minimally invasive TKA, but there is currently not enough evidence to support its preservation during open TKA.

In summary, the validity of this study may be undermined by the low initial response rate and patient retention rate, and gender-related differences in pain reporting. The effect of IPFP resection on post-TKA patients warrants further investigation via a large randomised controlled trial.

\section{Harrison X Bai, ${ }^{1}$ Ashley M Lee, ${ }^{1}$ Zhili Wang, ${ }^{2}$ Haiyun Tang, ${ }^{3}$ Li Yang ${ }^{2}$}

${ }^{1}$ Department of Neurology, The Second Xiangya Hospital, Central South University, Changsha, Hunan, China

${ }^{2}$ Department of Radiology, Hospital of the University of Pennsylvania, Philadelphia, Pennsylvania, USA

${ }^{3}$ Department of Radiology, Xiangya Hospital, Central South University, Changsha, Hunan, China

Correspondence to Li Yang, Department of Neurology, The Second Xiangya Hospital of Central South University, 139 Renmin Middle Road, Changsha, Hunan 410011, China; yangli762@gmail.com

Contributors $L Y$ contributed to drafting of the manuscript and revising it critically for important intellectual content; AML and ZW contributed to gathering data, the conception and design and analysis and interpretation of data; HXB and HT contributed to the conception and design and revision of the manuscript. All authors contributed to final approval of the manuscript submitted.

Funding This work was supported by the Natural Science Foundation of China (grant number 81301988) to LY.

Competing interests None declared.

\section{Patient consent Obtained.}

Provenance and peer review Not commissioned; internally peer reviewed.

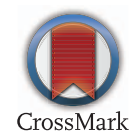

To cite Bai HX, Lee AM, Wang Z, et al. Ann Rheum Dis 2016;75:e3.

Received 17 September 2015

Accepted 19 September 2015

Published Online First 12 October 2015

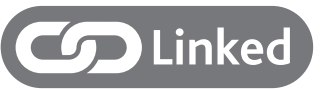

http://dx.doi.org/10.1136/annrheumdis-2015-208748

Ann Rheum Dis 2016;75:e3. doi:10.1136/annrheumdis-2015-208620

\section{REFERENCES}

1 Pan F, Han W, Wang $X$, et al. A longitudinal study of the association between infrapatellar fat pad maximal area and changes in knee symptoms and structure in older adults. Ann Rheum Dis 2015;74:1818-24.

2 Bartley EJ, Fillingim RB. Sex differences in pain: a brief review of clinical and experimental findings. $\mathrm{Br} J$ Anaesth 2013;111:52-8.

3 Fillingim RB, King CD, Ribeiro-Dasilva MC, et al. Sex, gender, and pain: a review of recent clinical and experimental findings. J Pain 2009;10:447-85.

4 Van Beeck A, Clockaerts S, Somville J, et al. Does infrapatellar fat pad resection in total knee arthroplasty impair clinical outcome? A systematic review. Knee 2013;20:226-31.

5 Pinsornsak P, Naratrikun K, Chumchuen S. The effect of infrapatellar fat pad excision on complications after minimally invasive TKA: a randomized controlled trial. Clin Orthop Relat Res 2014;472:695-701. 